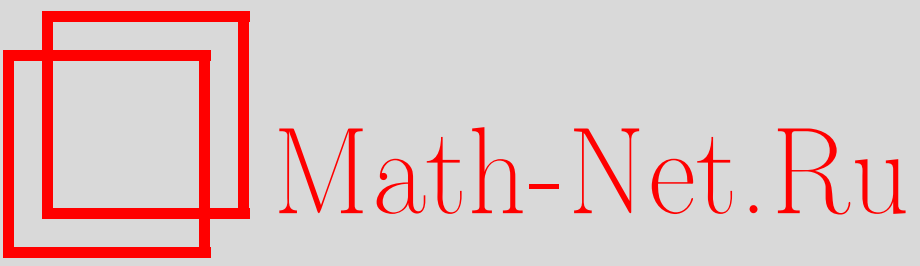

В. Ф. Тишкин, В. А. Гасилов, Н. В. Змитренко, П. А. Кучугов, М. Е. Ладонкина, Ю. А. Повещенко, Современные методы математического моделирования развития гидродинамических неустойчивостей и турбулентного перемешивания, Матем. моделирование, 2020, том 32, номер 8, 57-90

DOI: https://doi.org/10.20948/mm-2020-08-05

Использование Общероссийского математического портала Math-Net.Ru подразумевает, что вы прочитали и согласны с пользовательским соглашением

http://www.mathnet.ru/rus/agreement

Параметры загрузки:

IP : 54.147 .182 .235

26 апреля 2023 г., 12:18:06 


\section{СОВРЕМЕННЫЕ МЕТОДЫ \\ МАТЕМАТИЧЕСКОГО МОДЕЛИРОВАНИЯ \\ РАЗВИТИЯ ГИДРОДИНАМИЧЕСКИХ НЕУСТОЙЧИВОСТЕЙ И ТУРБУЛЕНТНОГО ПЕРЕМЕШИВАНИЯ}

\section{(C) 2020 2. $\quad$ В.Ф. Тишкин, В.А. Гасилов, Н.В. Змитренко, П.А. Кучугов, М.Е. Ладонкина, Ю.А. Повещенко}

Институт прикладной математики им. М.В. Келдыша РАН v.f.tishkin@mail.ru

Работа выполнена при финансовой поддержке РФФИ, грант №19-11-50130. ${ }^{1}$

DOI: $10.20948 / \mathrm{mm}-2020-08-05$

Изучение развития возмущений под действием различных гидродинамических неустойчивостей, а также переход к развитому перемешиванию и турбулентности, уже на протяжении многих десятилетий представляет значительный интерес. В первую очередь это связано с важностью этих процессов для различных областей науки и техники. Кроме того, следует отметить, что до сих пор не получено окончательных результатов, касающихся, например, характеристик турбулентных течений. Всё это стимулирует большой интерес к данной тематике как в плане физической теории, так и в плане развития новых подходов к математическому моделированию соответствующих задач. Возможности современной вычислительной техники позволяют проводить численные эксперименты как в двумерных, так и в трёхмерных постановках, анализировать особенности предлагаемых новых численных методов. В настоящее время на практике применяется огромное количество таких методов со многими их модификациями. Данный обзор посвящен наиболее перспективным, по мнению авторов, из них.

Ключевые слова: математическое моделирование, численные методы высокой точности, гидродинамические неустойчивости, турбулентное перемешивание.

\section{MODERN METHODS OF MATHEMATICAL MODELING OF THE DEVELOPMENT OF HYDRODYNAMIC INSTABILITIES AND TURBULENT MIXING}

\section{V.F. Tishkin, V.A. Gasilov, N.V. Zmitrenko, P.A. Kuchugov, M.E. Ladonkina, Y.A. Poveschenko}

Keldysh Institute for Applied Mathematics RAS, Moscow

\footnotetext{
${ }^{1}$ The reported study was funded by RFBR, project number 19-11-50130.
} 
The study of the development of perturbations under the influence of various hydrodynamic instabilities, as well as the transition to turbulent mixing and turbulence, has been a subject of considerable interest over the past decades. This is primarily due to the importance of these phenomena for various fields of science and technology. It should be noted, that the final results of the turbulent flows characteristics study have not yet been obtained. This fact stimulates a great interest in this topic, both in sense of physical theory and in sense of approaches to mathematical modeling and numerical methods development. The capabilities of modern computer technology make it possible to carry out numerical experiments in two-dimensional and three-dimensional settings, and to analyze the features of new numerical methods. To date, there are a huge number of methods and their modifications are applied in practice. This review is devoted to the most promising, according to the authors, of them.

Key words: mathematical modeling, high accuracy numerical methods, hydrodynamic instabilities, turbulent mixing.

\section{1. Общие замечания о методах численного решения газодинамических задач}

В настоящее время огромное количество работ посвящено исследованию развития гидродинамических неустойчивостей, возникающих в задачах астрофизики, инерционного термоядерного синтеза (ИТС), метеорологии, геофизики и многих других.

Исследование турбулентной стадии неустойчивостей стало возможно лишь в настоящее время в связи с бурным развитием многопроцессорной вычислительной техники [1]. Наличие высокопроизводительной техники позволило проводить трехмерное математическое моделирование развития гидродинамических неустойчивостей вплоть до автомодельной стадии турбулентности и получать физически оправданные результаты, а также качественно и количественно новые характеристики течений.

Существует несколько принципиально различных с физической точки зрения подходов к моделированию турбулентных течений. К ним относится прямое численное моделирование (ПЧМ, Direct Numerical Simulation, DNS), метод крупных вихрей (MKB, Large Eddy Simulation, LES), использование уравнений Рейнольдса для описания течений (Reynolds-averaged Navier-Stokes, RANS), неявные методы крупных вихрей MILES/ILES (Monotone Integrated LES/Implicit LES), а также гибридые подходы на основе перечисленных.

Прямое численное моделирование позволяет разрешать все пространственные и временные масштабы при моделировании процессов перемешивания и турбулентных течений. Однако, на сегодняшний день для практически интересных приложений с умеренными и высокими числами Рейнольдса та- 
кой подход практически не реализуем даже с учётом достижений вычислительной техники. Необходимость разрешать все масштабы вплоть до колмогоровского [2] $\left(\lambda_{0} \sim\left(v^{3} / \varepsilon\right)^{1 / 4}\right)$ приводит к оценке размера сетки $N^{3} \geq \operatorname{Re}^{9 / 4}$, которая превосходит возможности современных суперкомпьютеров. В связи с этим значительное развитие получили подходы, позволяющие существенно ослабить требования к разрешаемым масштабам, упомянутые выше. Тем не менее не все из них применимы при решении задач, например, ЛТС.

В некоторых случаях, когда наибольший интерес представляет изучение установившегося течения, для моделирования можно использовать усреднённые уравнения Навье-Стокса (RANS, уравнения Рейнольдса) [3-5]. Данные уравнения содержат члены, зависящие от пульсационных составляющих полей, для определения которых необходимо использовать различные модели турбулентности. Большинство турбулентных моделей сфокусировано на вычислении членов тензора напряжений Рейнольдса, вид которого в большинстве случаев записывается в соответствии приближением Буссинеска через тензор осреднённых скоростей деформации. Отметим, что возможно использование и других видов тензора напряжений Рейнольса (см., например, [6]. Значительно меньшее внимание уделяется другим членам, связанным с турбулентным переносом, молекулярной диффузией и турбулентным поток тепла, которые также необходимо определить для замыкания исходной системы уравнений.

Существует обширный круг различных моделей турбулентности как алгебраических, в которых необходимые величины типа турбулентной вязкости и турбулентной энергии записываются в явном виде, так и дифференциальных, в которых для их нахождения требуется решать дополнительные уравнения [4, 7-10]. На сегодняшний день созданы и используются сложные многопараметрические модели турбулентности, позволяющие учитывать анизотропию течений [11-14]. Однако следует отметить, что наличие столь большого числа разнообразных моделей турбулентности свидетельствует о достаточно узкой области применимости для каждой из них. Кроме того, большинство из них содержат полуэмпирические константы, которые в общем случае зависят от решаемой задачи и требуют корректировки в каждом конкретном случае.

Результатом моделирования с помощью RANS-подхода являются усреднённые поля, для которых влияние нестационарного турбулентного движения учтено через модель турбулентности. RANS-подход последние несколько десятилетий является основным для промышленных приложений, т.к. позволяет достаточно точно предсказывать осреднённые характеристики 
турбулентного взаимодействия и аэродинамические параметры исследуемой конфигурации при небольших вычислительных затратах, однако не позволяет изучать переходные быстро развивающиеся течения, характерные для задач физики высоких плотностей энергии.

Альтернативой методам RANS является метод крупных вихрей [15]. Традиционный МКВ заключается в использовании исходных уравнений Навье-Стокса с применением к ним фильтра, ограничивающего разрешаемые масштабы течения, и явной подсеточной (SGS, sub-gridscale) модели, позволяющей имитировать диссипацию энергии в мелких неразрешённых масштабах.

МКВ успешно применяется при моделировании большого числа модельных течений, однако имеет чрезмерную диссипацию при моделировании развития неустойчивостей, начиная с роста малых начальных возмущений и до развитого турбулентного течения $[16,17]$. Кроме того, конструирование подсеточных моделей для сжимаемых течений с резкими градиентами плотности и сильными разрывами, характерными для задачи физики высоких плотностей энергии, представляется затруднительным. Несмотря на это существуют примеры расчётов развития гидродинамических неустойчивостей на основе LES-подхода.

Структурное сходство уравнений, используемых в RANS и LES подходах позволяет комбинировать эти подходы, используя их положительные свойства. Гибридные подходы требуются в основном для моделирования отрывных турбулентных течений в задачах обтекания твердых тел. Одним из широко используемых гибридных RANS/LES методов является метод отсоединённых вихрей (Detached Eddy Simulation, DES) $[18,19]$. В этом случае выбор того или иного подхода (RANS или LES) к описанию турбулентности производится автоматически в зависимости от соотношения между локальным размером вычислительной сетки и характерным линейным масштабом турбулентности в соответствующей области. Использование уравнений Рейнольдса для описания течения в пограничном слое позволяет снять жёсткие ограничения на размеры ячеек сетки в пристенной области в направлении, параллельном стенке. DES подход демонстрирует высокую эффективность и экономичность. Однако остаются вопросы об адекватности результатов в области перехода от уравнений Рейнольдса к LES ("серая" область). Имеются и другие реализации комбинированных подходов, такие как метод ограниченных численных масштабов (Limited Numerica Scales, LNS), двухзонный подход (Zonal LES, ZLES) и другие. Достаточно подробное описание различных подходов на основе LES содержится в [20]. 
Моделирование подсеточной диссипации может быть обусловлено используемым численным методом - MILES/ILES-подход или неявный метод крупных вихрей $[21,22]$. В этом случае эволюцию крупных структур можно считать верной до тех пор, пока их масштаб не станет сопоставим с масштабом, определяемым схемной или физической вязкостью. Скорость диссипации на масштабах меньше порогового может быть обусловлена непосредственно численной диссипацией, либо недостатком или избытком вихрей для мелких масштабов. В идеальном случае скорость диссипации должна контролироваться явно, однако в этом случае будут наблюдаться сходные с LES-методами проблемы. Необходимо отметить, что использование ILES-подхода для моделирования перемешивания и турбулентных течений требует аккуратного выбора расчётной сетки и разностной схемы.

Применение того или иного численного метода не ограничивается выбранным подходом, однако подход будет определять существенные требования к свойствам схем. По этой причине дальнейшее обсуждение методик будем рассматривать в рамках ILES подхода.

Изучение имеющейся литературы по данной тематике позволяет утверждать, что на сегодняшний день существует огромное число различных численных методов и их модификаций, применяемых на практике, см., например, обзоры [20]. Обзор наиболее часто используемых из них для моделирования развития гидродинамических неустойчивостей и турбулентного перемешивания следует начать с некоторых общих положений.

Отправной точкой для большинства конструкций сеточных схем решения задач газовой динамики (ГД), магнитной газовой динамики (МГД), радиационной газовой динамики (РГД) и т.д. является запись исходных законов сохранения в виде:

$$
\frac{\partial \mathbf{U}}{\partial t}+\sum_{k=1}^{d} \frac{\partial \mathbf{F}_{k}}{\partial x_{k}}=\mathbf{S},
$$

где $\mathbf{U}$ - вектор неизвестных, $\mathbf{F}_{k}$ - вектор потоков вдоль координатных направлений, $\mathbf{S}$ - источники. Вектор $\mathbf{U}$ включает набор скаляров и векторов плотность, давление, скорость (магнитное поле в случае МГД), полную энергию газа, $\mathbf{F}_{k}$ представляет собой, соответственно, потоки массы, импульса, энергии, $d$ - размерность пространства. Система газодинамических уравнений должна быть замкнута дополнительным уравнением, а именно уравнением состояния, которое учитывает термодинамические свойства среды. В более сложных случаях, когда могут быть существенны диссипативные процессы, основная система (1) дополняется уравнениями этих про- 
цессов и выражениями для входящих в них коэффициентов и функций.

Обширную область газовой динамики составляют исследования течений газовых смесей. Для моделирования таких течений применяются специальные подходы, такие, например, как метод расчета концентраций в различных вариантах эйлеровых и лагранжевых методик [23,24]. В рамках односкоростной модели сплошной среды концентрации компонент газовой смеси рассчитываются путем решения соответствующих уравнений типа конвекции-диффузии, которые можно записать в такой же дивергентной форме, как и вышеприведенные уравнения системы (1). Течения веществ в разных фазовых состояниях (газодисперсных, парокапельных и т.д.), как правило, моделируются согласно концепции, в которой разные фазы характеризуются собственными атрибутами - полями плотности, давлений, температуры, скорости. Для каждой фазы записываются уравнения неразрывности, движения, сохранения энергии, а также дополнительное уравнение, описывающее эволюцию объемной концентрации фазы. Решаемая общая система уравнений может быть представлена в виде двух подсистем, гиперболической подсистемы и подсистемы уравнений релаксации давлений и скоростей фаз за счет их взаимодействии. Релаксация давления обусловлена микроскопическими движениями среды за волнами давления, а релаксация скорости определяется межфазным обменом импульсами. Основу расчетов многокомпонентных или многофазных течений составляют те же методы численного решения гиперболических или параболических уравнений, которые применяются и для численного анализа однородных газовых течений.

Отдельный интерес представляет решение системы (1), описывающей течения в сходящейся геометрии. Расчеты одномерных течений со сферической или цилиндрической симметрией, так же, как и двумерных осесимметричных течений, широко используются в различных прикладных исследованиях, когда можно сделать соответствующие упрощения в постановке задачи. Они также применяются для верификации новых численных методик и т.п. В связи с этим целесообразно отметить одно важное обстоятельство. Для решения таких задач газодинамические уравнения обычно записываются в сферических или цилиндрических системах координат и в этом случае содержат геометрические добавочные члены типа «источника», не содержащие дифференциальных слагаемых. Проблемы аппроксимации этих слагаемых обсуждались в ряде работ, например, в [25], в которых отмечается, что эта процедура требует специального внимания для обеспечения точности, особенно когда рассчитываются быстропротекающие процессы. 
Основу различных численных методов составляют сеточные, чаще всего, конечно-разностные аппроксимации системы уравнений (1). Основным современным подходом к аппроксимации уравнений в форме законов сохранения является метод конечных объёмов. При моделировании практически значимых задач применяются преимущественно аппроксимации членов дифференциальных уравнений не ниже второго порядка точности в области гладкого решения и, соответственно, TVD, ENO/WENO, PPM и другие реконструкции. Как известно, при использовании таких схем возникает необходимость применения монотонизирующих ограничителей потоков для подавления возникающих численных осцилляций. В настоящее время разрабатываются разнообразные новые типы монотонизирующих ограничителей потоков.

В последние два десятилетия особенно активно развивается метод Галеркина с разрывными базисными функциями (Discontinuous Galerkin Method, DGM), который обладает рядом достоинств, присущих как конечно-элементным, так и конечно-разностным аппроксимациям. Особенностью данного метода является компактность пространственного шаблона и при этом способность обеспечить заданный порядок точности на многомерных неструктурированных сетках с произвольной формой ячеек. Применение DGM позволяет использовать одновременно два подхода для повышения точности получаемого решения в областях существующих особенностей решения. Это повышение порядка точности метода за счет повышения порядка используемых полиномов, а также локальное измельчение сетки.

Метод опорных операторов $[26,27]$ используется для согласованной в смысле некоторых интегральных тождеств векторного анализа аппроксимации сопряжённых операций div, grad, rot и т.п. Это позволяет встроить в газодинамическую модель не только дивергентные законы сохранения, но и покомпонентное сохранение видов энергии в системе. В последние годы здесь построены и исследованы новые классы монотонизированных разностных схем второго порядка точности с использованием адаптивной искусственной вязкости численного решения уравнений газовой динамики.

Перспективным методом решения развития гидродинамической неустойчивости является схема «КАБАРЕ», основанная на схеме [28]. В [29,30] схема [28] была существенно переработана и фактически построен новый класс вычислительных алгоритмов, использующих две системы гидродинамических величин - одна для вычисления потоков в полуцелых точках и вторая - консервативные величины, обеспечивающие выполнение дискретных законов сохранения. 
К перспективным методам также относятся так называемые бикомпактные схемы [31]. Эти схемы четного (четвертого и выше) порядка пространственной аппроксимации конструируются с помощью интегро-интерполяционного подхода на минимальном шаблоне, занимающего одну пространственно-временную ячейку разностной сетки. Следствиями такого построения бикомпактных схем являются их консервативность [31,32], сохранение порядка аппроксимации на неравномерной конечно-разностной сетке и улучшенные дисперсионные свойства по сравнению с другими компактными схемами того же порядка аппроксимации [33].

В обзоре наряду с упомянутыми подходами также обсуждаются результаты анализа схем с искусственной вязкостью и методы повышения точности на адаптируемых сетках.

\section{2. Монотонизированные разностные схемы для уравнений в виде зако- нов сохранения}

Базой современных методов численного решения многомерных нестационарных задач газовой динамики с ударными волнами и разрывами других типов являются разностные схемы для систем одномерных нелинейных уравнений гиперболического типа. Их используют для изучения способов вычисления потоков на границах между ячейками в многомерных разностных схемах, подходов к монотонизации численного решения и многого другого $[34,35]$.

Как известно, решения задач математической физики, описываемых гиперболическими системами уравнений, могут быть гладкими в одних областях и разрывными в других. Разрывные решения могут при этом возникать из гладких начальных данных. Такие свойства решений налагают на алгоритмы численного решения гиперболических систем уравнений достаточно противоречивые требования. С одной стороны, численный метод должен сохранять свойство монотонности в тех областях, где искомые решения имеют большие перепады значений. С другой стороны, тот же метод должен обладать высоким порядком точности в тех областях, где решение является гладким. В [36] С.К. Годунов показал, что линейных разностных схем, удовлетворяющих этим двум требованиям, одновременно быть не может. Кроме того, была сформулирована монотонная схема Годунова, имеющая первый порядок и на каждом шаге которой в соседних ячейках решалась задача Римана о распаде разрыва. В дальнейшем это породило целый класс схем, называемыми схемами типа Годунова.

В [37-39] показано, что требование монотонности разностных производных решения накладывает на разностную схему жёсткие ограничения. 
Смысл этих работ заключается в конструировании разнообразных нелинейных алгоритмов, зачастую эвристического характера, которые обеспечивают непрерывный переход от немонотонной разностной схемы второго порядка аппроксимации к монотонной разностной схеме первого порядка аппроксимации с наветренными, или противопоточными аппроксимациями потоков в балансно-консервативных схемах. Разностные формулы повышенного порядка аппроксимации используются в точках, в которых численное решение является гладким, а в точках, в которых решение разрывное, используются монотонные схемы низкой точности. За последние несколько десятилетий эта ведущая идея получила значительное развитие, вследствие чего появилось большое количество подходов, зачастую сильно отличающихся друг от друга, опубликованных как у нас в стране, так и за рубежом. Здесь мы отметим только наиболее значительные разработки, получившие широкое распространение для прикладных работ.

В одной из первых работ по нелинейным схемам для решения газодинамических уравнений предлагается использовать комбинацию схем разного порядка точности, например, схем 1-го и 2-го порядков с переключением в зависимости от поведения решения [40].

Первая работа, в которой вместо комбинации схем использовалась оригинальная методика ограничения разностных производных при вычислении потоков через грани контрольного объема, была опубликована В.П. Колганом [41]. Основные этапы развития монотонизированных разностных схем повышенного порядка точности в 70-е годы за рубежом связаны с работами [42-44].

В этих схемах, известных как MUSCL (Monotonic Upwind Systems of Conservation Laws), для реализации метода Годунова используется линейная экстраполяция переменных на грань контрольного объема, через которую путем решения задачи о распаде разрыва вычисляются потоки, использующиеся затем в балансных уравнениях массы, импульса, энергии. Схема оказалась весьма удачной во многом благодаря предложенному ограничителю потоков типа minmod и получила широкое распространение.

Примерно в эти же годы большую популярность приобрёл метод антидиффузии с коррекцией потока или FCT-метод (Flux-Correcrted Transport) [45], после существенного его усовершенствования в [46].

Схемы с коррекцией потоков представляют собой двухэтапный алгоритм типа предиктор-корректор. Этап предиктора выполняется по монотонной схеме первого порядка, на втором этапе к схеме первого порядка добавляется ограниченная искусственная антидиффузионная добавка, повышаю- 
щая точность схемы в целом.

Двухэтапная разностная схема, предложенная в [47], реализует другой подход к коррекции потоков. В отличие от предыдущей описанной схемы, в ней на этапе предиктора выполняется расчёт шага по времени со вторым порядком аппроксимации, а затем анализируется результат и сглаживаются осцилляции. В ряде случаев данный подход дает более точные результаты, чем классическая коррекция потоков. Использование на первом этапе схемы второго порядка дает более полную информацию о решении. В алгоритме [45] сначала идет расчет по схеме низкого порядка, т.е. по схеме с большой схемной вязкостью. Таким образом, антидиффузионные поправки применяются к распределению, в котором физические максимумы и минимумы, если они были, сглажены на этапе предиктора, т.е. в расчете по схеме первого порядка, и информация о неоднородности решения к моменту корректора уже несколько утрачена.

Методы монотонизации численного решения зачастую критикуются за то, что с их помощью стремятся устранить нефизичные осцилляции в распределениях исходных величин, таких как скорости, плотности и т.П., которые могут быть немонотонны и содержать коротковолновые составляющие. При этом достаточно трудно отделить немонотонность физической природы от численной при использовании простого инструментария типа искусственной диффузии или антидиффузии.

В то же время уравнения газовой динамики удовлетворяют принципу максимума для инвариантов Римана, и многие современные схемы повышенной точности построены с учетом этого свойства. Метод коррекции потоков стал вытесняться более точными методиками. Дальнейшее продвижение в области разностных схем повышенной точности связано с работами [48-50], в которых был представлен метод, получивший название Total Variation Diminishing (TVD) или метод с невозрастающей полной вариацией. В данном подходе обеспечивается принцип максимума для характеристических переменных, а в физических переменных этот метод вместо монотонности не увеличивает полную вариацию, благодаря чему более точно передаёт характер поведения разрывных решений.

Общим во всех методах подобного класса является использование разнообразных монотонизирующих ограничителей потоков с переключателями, зависящими от локальных свойств численных решений. Схемы высокого порядка точности на основе решения задачи о распаде разрыва включают в себя различные ограничители потоков, от простого ограничителя типа minmod [42-44], до весьма сложных, с большим числом переключений, как, например, ограничитель superbee $[51,52]$. 
Кроме требования монотонности или требования неувеличения полной вариации, большое влияние на точность расчётов оказывают дополнительные требования, особенно требование неубывания энтропии [51,52]. Это требование необходимо тем или иным способом учитывать при построении разностных схем газовой динамики, чтобы не возникало ударных волн разрежения в звуковой точке при описании сверхзвуковых течений в переменных Эйлера. Это требование вводит усложнения в разностные схемы типа TVD и, тем самым, в конструкции ограничителей потоков, что подробно описано, например, в [51]. Подробное изложение основных проблем использования TVD-подхода для построения разностных схем газовой динамики и магнитной газодинамики дано в [35].

B последующем развитие схем типа TVD связано с разработкой новых монотонизирующих ограничителей потоков. Большинство предложенных ранее ограничителей имели дискретные переключатели, что приводит к разрывности первой производной и, тем самым, к снижению фактической точности. В [55-57] опробуются разнообразные гладкие ограничители.

Обобщением метода TVD явился метод реконструкции сеточных решений с автоматическим анализом гладкости численных решений, получивший название Essentially Non-Oscillatory (ENO) $[53,54]$ или существенно неосциллирующие схемы. В [58-60] описана техника автоматического анализа гладкости численного решения, что сводится к определению ячеек сетки, в которых находятся разрывы самой анализируемой сеточной функции, разрывы её первой производной (слабые разрывы) и разрывы производных более высокого порядка, в соответствии с требуемым порядком аппроксимации. В зависимости от локального уровня гладкости, для реконструкции сеточного решения используются интерполяционные многочлены соответствующего порядка. Реконструированные величины используются для вычисления потоков по граням ячеек. В $[59,60]$ описаны многомерные обобщения ENOсхем для ортогональных пространственных сеток.

Кроме техники ограничителей потоков, при построении монотонизированных разностных схем повышенного порядка аппроксимации для гиперболических законов сохранения широкое распространение получила техника монотонной или квазимонотонной реконструкции сеточных решений, получившая название методов взвешенной реконструкции численных решений WENO $[61,62]$. Для повышения порядка аппроксимации надо использовать вместо кусочно-постоянной реконструкции более точную реконструкцию. Примеры таких реконструкций даны в [63-66]. В [67] это требование реализуется при построении реконструирующего интерполяционного полинома 
на основе процедуры вариационного типа.

Из работ последнего десятилетия по схемам повышенной точности с реконструкцией решения в том или ином виде заслуживают внимания конструкции неосциллирующих схем контрольных объемов произвольной точности по пространству и времени для решения линейных гиперболических систем на неструктурированных сетках в двух и трех пространственных измерениях [68-72].

Ключевым моментом этих разработок является построение оператора реконструкции [73]. В [73,74] с целью реконструкции применяется иерархический ортогональный базис [75]. Реконструкция на основе [75] выполняется не в физических координатах, а в базовой системе координат, которая позволяет устранить эффекты масштабирования и, таким образом, улучшить обусловленность матриц реконструкции.

Особый класс конечно-объёмных методов для решения гиперболических систем уравнений представляют собой рёберно-ориентированные схемы. В отличие от традиционных схем высокого порядка [76], в рёберно-ориентированных схемах консервативные переменные определяются в сеточных узлах, а потоки, используемые для аппроксимации законов сохранения, вычисляются в серединах сеточных рёбер или диагоналей элементов. Эти схемы были предложены как обобщение схем MUSCL на неструктурированные сетки. Современное состояние этих схем представлено, главным образом, схемами на основе метода коррекции потоков и схемами с квазиодномерной реконструкцией переменных Edge-BasedReconstruction (EBR) [77,78]. Схемы EBR на произвольных неструктурированных сетках обладают первым порядком аппроксимации в интегральных нормах, однако на регулярных сетках вырождаются в конечно-разностные схемы высокого порядка, что обеспечивает неплохое качество счёта в сравнении с другими схемами первого порядка аппроксимации.

Конструкции монотонизированных разностных схем чаще всего опираются на базовую монотонную разностную схему первого порядка аппроксимации, а именно на схему С.К. Годунова [79], для реализации которой требуется в каждой точке численно решать задачу о распаде разрыва. Наряду с развитием схем годуновского типа развивались и другие более простые для реализации монотонные или квазимонотонные разностные схемы. Начало этого направления положено в [80]. Метод Лакса-Фридрихса можно рассматривать в качестве альтернативы схеме Годунова, которая позволяет избежать решения задачи Римана на каждой грани ячейки за счет добавления искусственной вязкости. Эта схема довольно много использовалась в газодинамических расчетах благодаря неплохой точности и простоте реали- 
зации, однако она обладает заметной схемной вязкостью, которая в ряде случаев оказывается избыточно большой.

Потоки Лакса-Фридрихса-Русанова [81,82] для линейных гиперболических уравнений $\partial U / \partial t+A(\partial U / \partial x)=0$ отличается от потока Годунова тем, что в потоке Годунова $F_{i+1 / 2}^{\text {Godunov }}=A\left(U_{i+1}+U_{i}\right) / 2-L|\Lambda| L^{-1}\left(U_{i+1}-U_{i}\right) / 2$ используется матрица $L|\Lambda| L^{-1}$, где $L$ - матрица из левых собственных векторов матрицы $A$, а $\Lambda$ - матрица собственных значений, а в потоке Лакса-Фридрихса-Русанова $F_{i+1 / 2}^{L F R}=A\left(U_{i+1}+U_{i}\right) / 2-D\left(U_{i+1}-U_{i}\right) / 2$, где $D=C E$, где $C=\max _{i}\left|\lambda_{i}\right|$. Таким образом, видно, что диссипация схем Лакса-ФридрихсаРусанова превосходит диссипацию схем Годунова.

В [83-88] построены семейства центрально-разностных неосциллирующих схем высокого уровня разрешения для численного решения уравнений ГД, МГД и др. в одно-, дву- и трехмерном случаях, на регулярных и неструктурированных сетках. Достоинство схем с центральной аппроксимацией в том, что в них не используется в явной форме информация о волновой структуре потока и фактически явно не вычисляется якобиан матрицы линеаризованной системы уравнений, в то же время отсутствует необходимость расщепления потоков по направлениям. Для решения прикладных задач эти методики используются менее активно, чем методы на основе решения задачи Римана. Отчасти это может быть связано с проблемами и трудностями реализации таких схем на неравномерных прямоугольных сетках и обобщением на неструктурированные сетки.

В целом можно сказать, что в последнее десятилетие при построении разнообразных разностных схем для гиперболических законов сохранения использование монотонизирующих ограничителей потоков стало общепринятой практикой. В практических исследованиях стали применяться преимущественно аппроксимации переменной или адаптивной точности не хуже второго порядка точности в области гладкого решения. Аппроксимации первого порядка практически выходят из употребления. Совместно со схемами типа TVD, ENO/WENO для аппроксимации по времени используются методы Рунге-Кутты третьего и четвёртого порядков точности.

\section{3. Метод аппроксимации конвективных потоков на основе кусочно-па- раболической интерполяции}

Одной из наиболее удачных разработок монотонизированных схем повышенной точности для моделирования газодинамических процессов с нестационарными ударными волнами является кусочно-параболический метод (РPM - Piecewise Parabolic Method, предложенный в [89,90]). В PPM-ме- 
тоде, наряду с реализацией условий для устранения нефизичных экстремумов, в алгоритм реконструкции решения введена процедура уменьшения численной диффузии, влияние которой на контактные разрывы в численном решении делает их более резкими. В публикации специалистов Ливерморской национальной лаборатории США [91] представлены результаты моделирования неустойчивости Рихтмайера-Мешкова, переходящей в турбулентность, на сетке порядка 10 млрд ячеек, и делается вывод о том, что РРМ имеет хорошую перспективу для прямого трёхмерного моделирования турбулентности. Разумеется, это возможно только при надлежащем уровне развития массивно-параллельных вычислительных систем. Возможности воспроизведения достаточно тонкой структуры течений с помощью РРМ показаны в [91-93] при сравнении результатов моделирования дифракции ударной волны на клине с данными экспериментов. Учитывая преимущества кусочно-параболического метода, заключающиеся в его универсальности, экономичности и точности аппроксимации, он используется в качестве базового в таких программных комплексах, как ЭГАК и ТРЭК, использующихся в РФЯЦ ВНИИЭФ [94].

Перспективной модификацией РРМ является кусочно-параболический метод на локальном шаблоне (Piecewise Parabolic Methodona Localstencil PPML). Метод PPML является оригинальной разработкой для решения уравнений гиперболического типа в виде законов сохранения, развитой в астрофизической группе ИПМ им. М.В. Келдыша РАН. Работы этой группы убедительно доказали эффективность PPML на ряде астрофизических задач и получили международное признание [95]. Метод PPML имеет третий порядок точности по пространству и второй по времени. Основы метода и некоторые результаты его приложения к задачам астрофизики изложены в серии публикаций [96-98].

Метод PPML предназначен для широкого круга задач моделирования газовых течений, в том числе с магнитными полями, и представляет собой способ реконструкции решения внутри разностных ячеек с использованием кусочных парабол, строящихся по трем точкам вдоль каждого координатного направления. Одна из таких точек соотносится с центром ячейки, две другие - с границами ячейки в соответствующем направлении. PPML близок к РРМ в формулировке $[89,90]$, однако имеет существенное отличие, состоящее в способе вычисления значений на границах ячеек. В классическом методе РРМ граничные значения вычисляются с помощью интерполяционной процедуры на расширенном четырехточечном шаблоне. В методе PPML такая процедура исключена и вместо нее используется характеристическая 
интерполяционная процедура внутри ячейки (на компактном шаблоне) с предыдущего временного слоя на текущий.

В исходном варианте метода РРМ четырехточечная интерполяция приводит к излишней диссипации, например, на контактном разрыве, и, как следствие, к необходимости применения специальной подстройки интерполяции для уменьшения сглаживания фронтов разрывов. Кроме того, возникают вычислительные проблемы при построении и использовании корректных граничных условий в расчетной области. Интерполяция на расширенном шаблоне обеспечивает высокое качество результатов на гладких решениях, но может давать осцилляции на разрывах. Использование нелинейных механизмов стабилизации решения, таких как ограничители потоков, или “лимитеры”, решает проблему осцилляций на разрывах, однако тогда возникают проблемы в задачах с физически обусловленными осциллирующими решениями, т.к. диффузия, обусловленная действием лимитеров, приводит к затуханию амплитуды осцилляций. При моделировании волн в средах с дисперсией действие лимитеров может приводить к неправильным результатам в картине развития неустойчивости.

В методе PPML устранены перечисленные недостатки метода PPM. Ocновная идея PPML состоит в учете локального поведения характеристик в потоке газа. При построении интерполяций с целью вычисления гидродинамических параметров на границах контрольного объема и, соответственно, потоков, используются инварианты Римана, переносимые вдоль характеристик с предыдущего слоя по времени. Для применения характеристического анализа в модифицированном методе РРМ в разностных ячейках локально строится базис собственных векторов якобиана исходной нелинейной системы. После этого нелинейная задача определения потоков на границах контрольного объема линеаризуется в окрестности каждой границы ячейки, что приводит к системе «расщепленных» уравнений переноса. Состояния, вычисленные на границах ячеек с помощью локально-характеристического подхода к переносу амплитуд волн, в дальнейшем используются для построения кусочно-параболической интерполяции. При этом к амплитудам волн применяется процедура монотонизации, которая сохраняет порядок схемы в точках локального экстремума $[99,100]$. Для сохранения монотонности в многомерном в уравнении для амплитуд волн удерживаются члены, содержащие производные в тангенциальных направлениях по отношению к рассматриваемым границам ячеек. Это позволяет вычислить состояния для решения задачи Римана и реконструированные граничные величины, необходимые для построения кусочных парабол с учетом всех пространственных направлений. 
Прикладное программное обеспечение, созданное в ИПМ им. М.В. Келдыша PAH на основе PPML, было протестировано на серии тестовых задач. Моделировалось развитие неустойчивости Релея-Тейлора в одномодовом и многомодовом режимах, неустойчивость Рихтмайера-Мешкова, распространение взрывных ударных волн, направленный внутрь центральный взрыв, распад контактных разрывов и т.д. PPML при сохранении всех качеств метода РРМ обладает преимуществом по точности за счет меньшей численной диссипации.

Программный пакет PPML продемонстрировал уникальную точность расчета с идеальным сохранением симметрии решения для случая симметричных начальных данных. Сравнительный анализ тестовых расчетов, в котором использовались зарубежные «астрофизические» коды Athena и CASTRO показал превосходство кода на основе PPML. Соответствующее обсуждение можно найти в [101]. Так, например, посредством этого кода был промоделирован взрыв кислородного ядра звезды с массой в сто раз больше солнечной. Получено высокое качество воспроизведения динамики ударных волн, в том числе показано их расщепление. Расчетным путем обнаружена внутренняя конвективная зона, что является следствием весьма тонких эффектов, которые ранее не воспроизводились в подобных расчетах.

\section{4. Метод Галеркина с разрывными базисными функциями}

В последние два десятилетия особенно активно развивается метод Галеркина с разрывными базисными функциями (РМГ) или Discontinuous Galerkin Method (DGM), первое упоминание о котором в [102]. Ежегодно появляется огромное количество работ, посвященных исследованию и применению разрывного метода Галеркина. На одной из конференций в рамках ECCOMAS [103] более половины докладов были посвящены применению разрывного метода Галеркина.

DGM относится к численные методам высокой точности. Данный метод обладает рядом достоинств, присущих как конечно-элементным, так и конечно-разностным аппроксимациям. Его особенностью является компактность пространственного шаблона и при этом способность обеспечить заданный порядок точности на многомерных неструктурированных сетках с произвольной формой ячеек [104]. Это особенно актуально для решения сложных разномасштабных задач. Как известно, существует два подхода для повышения точности получаемого решения. Один из них - измельчение сетки в областях существующих особенностей решения, второй подход повышение порядка точности схемы. Применение разрывного метода Галеркина позволяет использовать сразу оба подхода как повышение порядка 
точности метода, за счет повышения порядка используемых полиномов, так и локальное измельчение сетки (так называемая $h p$-адаптация) [105-107].

При решении уравнения Навье-Стокса разрывным методом Галеркина, уравнения записываются в виде системы уравнений первого порядка и решение происходит в два этапа [108]. На первом этапе вычисляются компоненты градиента температуры и тензора вязких напряжений, аппроксимация которых, как и аппроксимация решения, в пределах ячейки сетки при реализации модального подхода находится в виде полиномов степени $p$ с зависящими от времени коэффициентами. На границе элемента потоковые значения величин определяются по некоторому правилу от значений внутри элемента и от значений в соседней к данному элементу ячейке.

На следующем этапе определяются компоненты вектора консервативных переменных. При этом конвективные потоки могут быть рассчитаны при помощи различных вариантов точного или приближенного решения задачи Римана, диффузионные потоки на границе элемента также могут быть рассчитаны различными способами, подробный анализ которых проведен в [109]. Для вычисления интегралов используются квадратурные формулы нужного порядка. Данный двухэтапный подход позволяет вычислять градиенты с тем же порядком точности, что и консервативные переменные, сохраняя компактность шаблона схемы.

Одним из важных вопросов при реализации метода является выбор сетки, на которой ищется решение. Несомненным преимуществом DGM является возможность его применения на сетках произвольной структуры. В настоящее время разрывный метод Галеркина хорошо разработан как для структурированных [110], так и для неструктурированных [111] сеток. Существуют удачные программные реализации DGM для решения трехмерных задач на неструктурированных сетках, содержащих как элементы только одного типа - тетраэдральные [111-114] или гексаэдральные [105], а также для сеток произвольной структуры [115]. Очевидным недостатком метода является его чрезвычайно высокая вычислительная стоимость, но это покрывается его компактным шаблоном и, следовательно, созданием эффективных параллельных программных комплексов.

Как известно, наряду с множеством преимуществ применения разрывного метода Галеркина, он имеет некоторые сложности реализации. Во-первых, для обеспечения монотонности решения, полученного данным методом, необходимо вводить так называемые ограничители наклона, или лимитеры, в особенности в том случае, если решение содержит сильные разрывы. Наиболее широко используемым, является лимитер Кокбурна [116]. 
Идея данного лимитера легко реализуется в многомерном случае на сетках произвольной структуры. Однако данный лимитер, как и все TVD лимитеры, снижает точность получаемого решения. В последнее время активно развиваются различные подходы к решению этой проблемы. Один из подходов к созданию лимитера повышенного порядка точности предложен в работах Криводоновой [117]. Но данный лимитер хорошо работает только на структурированных сетках. Другие подходы к созданию лимитеров повышенного порядка точности изложены в [118-125].

Также важным вопросом при реализации DGM является вопрос о выборе дискретной аппроксимации потоков для вязких членов уравнения Навье-Стокса. Существует несколько видов таких аппроксимаций, наиболее часто используемых в реальных расчетах $[108,126]$, которые были исследованы в [127]. Однако вопрос оптимального выбора таких аппроксимаций остается открытым.

Как известно, при повышении порядка точности схемы, возникает жесткое ограничение на шаг по времени. Первоначально при расчетах DGM интегрирование по времени проводилось явными многошаговыми схемами Рунге-Кутты высокого порядка $[116,128,129]$. Но наиболее эффективный подход заключается в использовании и неявных методов интегрирования по времени с целью ослабления ограничения на шаг по времени [130-135].

B настоящий момент известны программные реализации DGM неявным методом для моделирования несжимаемых течений [103] и для решения уравнений Навье-Стокса [134].

Таким образом, для успешной реализации DGM на трехмерных неструктурированных сетках необходимо сосредоточить внимание на нескольких моментах. Во-первых, на построении лимитирующих функций, во-вторых, на выборе наилучших дискретных аппроксимаций диффузионных потоков, и, наконец, на применении неявных и итерационных методов решения полученных дифференциально-разностных уравнений.

Как известно, DGM обладает существенной вычислительной сложностью, поэтому встает вопрос о максимально эффективном использовании всех возможностей вычислительной техники. В настоящее время в мировых исследовательских центрах, занимающихся этой проблемой, ведутся работы по распараллеливанию реализаций DGM на суперЭВМ [136-138]. В [124] при решении уравнений Навье-Стокса DGM использован новый сеточно-операторный подход к программированию задач математической физики, позволяющий компактно записывать и эффективно реализовывать математические формулы, единообразно реализовывать подход на разных ти- 
пах сеток и для различных вычислительных архитектур, в том числе для графических ускорителей CUDA [113, 139, 140].

В [141] сравниваются DGM и метод конечного объема (FV) на декартовых сетках, а также на произвольных неподвижных и движущихся сетках Вороного. В данной работе DGM показал преимущества по сравнению с методом FV как на статических, так и на подвижных сетках. На различных тестовых задачах авторами продемонстрировано, что модификация DGM обеспечивает улучшенную точность по сравнению с подходами FV того же порядка, а также уменьшает осцилляции и искусственную диффузию.

\section{5. Схемы с искусственной вязкостью.}

Разностные схемы, в которых устранение нефизичных достигается путем добавки к давлению члена с искусственной вязкостью, до сих пор достаточно успешно используются в вычислительной практике, например, для решения различных задач радиационной плазмодинамики [142].

Хорошие результаты, продемонстрированные в цитированных работах, достигаются во многом благодаря тому, что, несмотря на необходимость подстройки параметров искусственной вязкости к задачам определенного круга, эта подстройка не создает больших проблем и достигается на основе априорных оценок и небольшого числа предварительных расчетов. Искусственная вязкость - широко распространенный прием в расчетах по «лагранжевым» схемам. Однако в целом прием сквозного счета течений с сильными неоднородностями (разрывами) путем добавления в уравнения движения добавки к давлению, аналогичной «второй вязкости», развивается отдельными специалистами и в настоящее время мало популярна. Более перспективными для решения трехмерных задач представляются методы, основанные на идеях «тензорной» вязкости, скорректированной с учетом невозрастания полной вариации решения [143].

Работа [144] явилась одной из первых в которых развивалась методика на основе искусственной тензорной вязкости применительно к лагранжевым разностным схемам расчета двумерных газодинамических течений.

Несмотря на солидный возраст разработок метода «искусственной вязкости», схемы с искусственной вязкостью адаптивного типа [145], в том числе тензорной, могут иметь перспективу в изучении газодинамических процессов с сопутствующими процессами (ионизация, радиационный перенос), т.к. в этом случае фронт волны (двухтемпературная плазма, сильно излучающие сверхкритические волны) уже не совсем такой, как в классическом случае [144, 146].

Механизм искусственной вязкости можно рассматривать как способ 
регуляризации разностной схемы с целью получения нелинейно-устойчивого решения. В связи с этим можно отметить перспективное направление схем с регуляризаторами, получаемыми на основе метода кинетического согласования. Кинетически-согласованная схема газовой динамики предложена Б.Н. Четверушкиным [147] и развивается в его работах, работах его коллег и учеников. Эти схемы строятся путем осреднения разностной схемы, аппроксимирующей уравнение Больцмана. В итоге осреднения получаются уравнения газовой динамики с некоторыми добавками, которые можно представить как некую регуляризацию схемы газодинамики. Кроме того, есть варианты «гиперболизированных» кинетически-согласованных схем, пригодные для расчетов вязких течений с явной аппроксимацией по времени, что удобно для распараллеливания [147]. Здесь вязкость трактуется как регуляризатор, стабилизирующий расчеты на сетках весьма высокой размерности (миллиарды ячеек).

В ряде работ искусственная вязкость, вслед за [144], применяется в виде, ориентированном по направлению движения ударной волны. Искусственная вязкость «тензорного типа» применяется сравнительно редко, но известно, что этот прием заметно улучшает качество расчётов, в том числе, выполняемых на неструктурированных сетках [148].

В.Ф. Куропатенко в 1962 г. предложил [149] разностную схему для лагранжевой газовой динамики на разнесенных сетках, которая использует метод С.К. Годунова [36] для определения коэффициента искусственной вязкости. Получающаяся при этом схема близка к схеме С.К. Годунова.

Для улучшения точности Христенсен [150] предложил уменьшать искусственную вязкость специальными множителями, заимствованными из методов типа FCT и TVD. Эти множители использовались в монотонизированных разностных схемах повышенного порядка аппроксимации для ограничения потоков на не разнесенных сетках.

В 1990-х годах зарубежными и отечественными специалистами были выполнены интересные разработки лагранжевых разностных схем на разнесенных сетках, в которых применены принципы построения монотонизированных разностных схем типа TVD [150,151]. За основу принимались как схема Годунова [150], так и центральная схема [151]. Во всех этих методиках монотонизация по принципу TVD выполнялась в форме модификации искусственной вязкости путем специальных множителей.

Монотонизация эйлеровых полностью консервативных разностных схем второго порядка точности может быть выполнена различными способами, в том числе посредством использования адаптивной искусственной вязкости 
для численного решения уравнений газовой динамики. В $[152,145]$ для уравнений газовой динамики в эйлеровых переменных построено семейство двухслойных по времени полностью консервативных разностных схем (ПКРС) с профилированными по пространству временными весами. Значительное внимание в работе уделено способам конструирования регуляризирующих потоков массы, импульса и внутренней энергии, не нарушающих свойств полной консервативности разностных схем данного класса, анализу их амплитуды и возможности их использования на неравномерных сетках. Разработанные схемы могут быть использованы для расчета процессов в неравновесных по температуре средах, например, при необходимости учета электрон-ионной релаксации температуры в короткоживущей плазме в условиях интенсивного энерговклада. Расчеты проводились с адаптивным превышением коэффициента линейной вязкости вида $\beta h^{2} / \tau$, где $\beta$ превышало число Куранта, являющееся в данном случае нижним пределом теоретической устойчивости процессов переноса, обеспечивающим наветренную (upwind) аппроксимацию. Высокочастотные нефизичные осцилляции минимизировались при задаваемом предельном локальном вязком превышении $\beta_{0}$, если они не исчезали заранее в процессе накопления этого вязкого превышения $\beta$ самим алгоритмом. Эти схемы оказались не дорогостоящими в вычислительном отношении и обеспечили, в частности, численное решение известной задачи Эйнфельда без выраженных энтропийных пиков в профилях температуры.

\section{6. Методы повышения точности расчётов на основе динамически адап- тируемых сеток}

Для повышения точности расчётов применяются методы двух основных направлений. Один основан на явном выделении областей больших градиентов (скачков) решения и подстройки подвижной сетки к особенностям структуры течения на основе анализа погрешности решения, принципов равнораспределения численных ошибок и т.д. При этом часто налагается условие сохранения числа узлов (ячеек) [153-158].

Другой, более распространенный метод адаптации сетки AMR (Adaptive mesh refinement) реализуется путем дробления/слияния ячеек, что более удобно для расчетов трехмерных многомасштабных течений по сравнению с методами с явным выделением разрывов. AMR специально разрабатывался для тех задач, в которых рассматриваются сильно различающиеся по масштабам структуры течения, нестационарные волновые процессы и т.п. AMR активно развивается за счет сочетания в соответствующих алгоритмах динамической адаптации сеток и схем повышенной точности. Сравнение методов $r$-уточнения и $h$-уточнения для установившихся трансзвуковых те- 
чений дано в [159]

Метод AMR работает в определенных зонах с тонкой, вихревой структурой, в зонах турбулентного перемешивая слоев и т.д. Процедура адаптации осуществляется динамически в течение всего времени расчета.

Техника настройки точности вычислений в соответствии со специфическими требованиями стала распространенной благодаря работам М. Бергер, И. Олигера и Ф. Колеллы $[160,93]$. Позже были выполнены многочисленные работы, подтверждающие высокие качества метода и показывающие разнообразие его возможных применений: от проблем турбулентного перемешивания до изучения многомасштабных структур в астрофизических объектах $[93,161]$. Возможности уточнения сеток могут быть расширены за счет использования функционалов от сеточных функций, позволяющих контролировать качество решения [162]. Динамическая адаптация сетки может использоваться и для расчетов стационарных течений методом установления.

Одними из основных сложностей в реализации данного подхода являются: выбор анализатора гладкости решения или критерия адаптации сетки, способы организации и хранения данных, а также методы балансировки параллельных алгоритмов.

Различные характеристики потока (например, ударные волны, сдвиги, контактные разрывы) проявляются в решении различными способами. Так что критерий, который хорошо подходит для ударных волн (например, градиент давления), может плохо подходить для сдвиговых течений. Одно из возможных решений этой дилеммы состоит в разработке универсального критерия, определяющего как можно больше особенностей. В работе [163], посвященной численному моделированию на адаптивных сетках взаимодействия ударных волн с пограничными слоями, проблема выбора критерия адаптации сетки решается основе вейвлет-анализа [164].

Другая проблема, которая возникает при реализации AMR подхода, заключается в том, что критерий обнаружения может привести к чрезмерному измельчению сетки, которая уже достаточно уточнена, в то время как области с некоторыми особенностями течения, так же нуждающиеся в уточнении, останутся незамеченными. В [165] дано отличное описание этой проблемы и предложен способ ее решения.

Подробный анализ различных проблем, возникающих при реализации AMR подхода проведен в [166].

B [167] схема AMR применяется при использовании LES модели турбулентных потоков с целью достижения достаточной разрешимости для мелких структур. Особое внимание уделено структуре хранения и организа- 
ции связей ячеек. А также применению методов AMR вместе с параллельными алгоритмами для разделения и балансировки вычислительной сетки.

Одним из примеров современного уровня моделирования можно привести работу [168], в которой авторы объединяют высокоточные численные схемы, улучшенную модель турбулентности (DDES)(SA) и AMR подход адаптации сеток характеристикам течения. Проводится численное моделирования с высоким пространственным разрешением вихревых неустойчивых потоков с отрывным течением в задачах обтекания цилиндра потоком с высоким числом Рейнольдса и двухлопастного винта вертолета. Обсуждаются вопросы эффективной параллельной реализации алгоритмов и вопросы балансировки нагрузки.

Сопоставление актуальных численных методик отражено в $[169,170]$, где на примере моделирования НРТ и НРМ проводится анализ интегральных характеристик зоны перемешивания, а также сравнение спектральных характеристик турбулентных течений.

\section{7. Заключение}

В настоящее время существует большое количество численных методов различного вида, применяемых для исследования развития гидродинамических неустойчивостей. Выбор того или иного метода определяется задачей, для которой необходимо провести моделирование, а также целью исследования. Так, при исследовании перехода к развитому перемешиванию и турбулентности определяющим фактором является низкая численная диссипация метода, позволяющая корректно восстанавливать спектр турбулентных пульсаций. В случае течений многокомпонентных смесей важным свойством используемого метода является низкая численная диффузия. Для численного моделирования течений с УВ иногда достаточно эффективным является применение более простых методов, восстанавливающих порядок точности за фронтом ударной волны.

\section{СПИСОК ЛИТЕРАТУРЫ}

1. Б.Н. Четверушкин, В.Ф. Тишкин. Применение высокопроизводительных многопроцессорных вычислений в газовой динамике / Математическое моделирование: проблемы и результаты. - М.: Наука, 2003, с.123-168;

B.N. Chetverushkin, V.F. Tishkin. Primenenie vysokoproizvoditelnyx mnogoprocessornyx vychislenij v gazovoj dinamike / Matematicheskoe modelirovanie problemy: i rezultaty. M.: Nauka, 2003, s.123-168.

2. А.Н. Колмогоров. Локальная структура турбулентности в несжимаемой вязкой жидкости при очень больших числах Рейнольдса // Докл. АН СССР, 1941, 30, №4, с.299-303; 
A.N. Kolmogorov. Lokalnaya struktura turbulentnosti v neszhimaemoj vyazkoj zhidkosti pri ochen bolshikh chislakh Rejnoldsa // Dokl. AN SSSR, 1941, 30, №4, s.299-303.

3. T.B. Gatski, J.-P. Bonnet. Compressibility, Turbulence and High Speed Flow, 2009.

4. D.C. Wilcox. Turbulence Modeling for CFD, 2006.

5. C. Hirsch. Numerical Computation of Internal and External Flows // Comp. Methods for Inviscid and Viscous Flows, 1990, v.2.

6. A.V. Rodionov. On the use of Boussinesq approximation in turbulent supersonic jet modeling // Int. J. Heat and Mass Transport, 2010, 53, №5-6, p.889-901.

7. P. Spalart, S. Allmaras. A one-equation turbulence model for aerodynamics flows // 30th Aerospace Sciences Meeting and Exhibit, 1992, Reno, NV, USA.

8. D.C. Wilcox. Formulation of the k- $\omega$ Turbulence Model Revisited //AIAA Journal, 2008, v.46, №11, p.2823-2838.

9. K. Hanjalic, B. Launder. A Reynolds stress model of turbulence and its application to thin shear flows // J. of Fluid Mech., 1972, 52, №4, p.609-638.

10. F.R. Menter. Two-Equation Eddy-Viscosity Turbulence Models for Engineering Applications // AIAA Journal, 1994, v.32, №8, p.1598-1605.

11. B.E. Morgan, M.E. Wickett. Three-equation model for the self-similar growth of RayleighTaylor and Richtmyer-Meshkov instabilities // Phys. Rev. E, 2015, v.91, №4, p.043002.

12. B.E. Morgan, O. Schilling, T.A. Hartland. Two-length-scale turbulence model for self-similar buoyancy-, shock-, and shear-driven mixing // Phys. Rev. E, 2018, v.97, №1, p.013104.

13. P.Y. Chou. On velocity correlations and the solutions of the equations of turbulent fluctuations // Quarterly of Appl. Math., 1945, v.3, №1, p.38-54.

14. J. Rotta. Statistische Theorie nichthomogener Turbulenz // Zeitschriftfür Physik, 1951, v.129, №6, p.547-572.

15. J. Smagorinsky. General circulation experiments with the primitive equations I. The basic experiment // Mon. Weather Rev., 1963, v.91, №3, p.99-164.

16. M. Lesieur, O. Metais. New trends in large-eddy simulations of turbulence // Ann. Rev. Fluid. Mech., 1996, №28, p.45-82.

17. S.B. Pope. Turbulent flows, 2000.

18. P.R. Spalart, W.-H. Jou, M. Stretlets and S.R. Allmaras. Comments on the Feasibility of LES for Wings and on the Hybrid RANS/LES Approach, Advances in DNS/LES, Proceedings of the First AFOSR International Conference on DNS/LES, 1997.

19. M. Strelets. Detached Eddy Simulation of Massively Separated Flows // AIAA 2001, p.2001-0879.

20. К.Н. Волков, В.Н. Емельянов. Моделирование крупных вихрей в расчётах турбулентных течений. - М.: Физматлит, 2008;

K.N. Volkov, V.N. Emelyanov. Modelirovanie krupnykh vikhrej v raschyotakh turbulentnykh techenij. - M.: Fizmatlit, 2008.

21. F.F. Grinstein, A.A. Gowardhan, J.R. Ristorcelli. Implicit large-eddy simulation of shockdriven material mixing // Philos. Trans. A Math. Phys. Eng. Sci., 2003, №371, 20120217.

22. F.F. Grinstein, L.G. Margolin, W.J. Rider (Eds.). Implicit Large Eddy Simulation: Computing Turbulent Fluid Dynamics, 2010.

23. С.М. Бахрах и др. Расчет газодинамических течений на основе метода концентраций // ДАН СССР, 1981, т.257, №3, с.566-569;

S.M. Bakhrakh $i d r$. Raschet gazodinamicheskikh techenij na osnove metoda koncentracij 
// DAN SSSR, 1981, t.257, №3, s.566-569.

24. С.М. Бахрах, В.Ф. Спиридонов. Метод концентраций расчета нестационарных течений сплошной среды // ВАНТ. Сер. Мат. мод. физ. процессов, 1999, №4, с.32-36;

S.M. Bakhrakh, V.F. Spiridonov. Metod koncentracij rascheta nestacionarnykh techenij sploshnoj sredy // VANT. Ser. Mat. mod. fiz. processov, 1999, №4, s.32-36.

25. A. Mignone. High-order conservative reconstruction schemes for finite volume methods in cylindrical and spherical coordinates // J. of Comp. Physics, 2014, №270, p.784-814.

26. А.А. Самарский, В.Ф. Тишкин, А.П. Фаворский, М.Ю. Шашков. Операторные разностные схемы // Дифф. уравнения, 1981, 17, №7, с.1317-1327;

A.A. Samarskij, V.F. Tishkin, A.P. Favorskij, M.Yu. Shashkov. Operatornye raznostnye skhemy // Diff. uravneniya, 1981, 17, №7, s.1317-1327.

27. K. Lipnikov, G. Manzini, M. Shashkov. Mimetic finite difference method // J. of Comp. Physics 2014, №257, p.1163-1227.

28. A. Iserles. Generalized leapfrog methods // IMAJ. Numer. Anal., 1986, v.6, № 3, p.381-392.

29. В.М. Головизнин, А.А. Самарский. Разностная аппроксимация конвективного переноса с пространственным расщеплением временной производной // Матем. Мод., 1998, т.10, №1, c.86-100;

V.M. Goloviznin, A.A. Samarskij. Raznostnaya approksimaciya konvektivnogo perenosa s prostranstvennym rasshchepleniem vremennoj proizvodnoj // Matem. Mod., 1998, t.10, №1, s.86-100.

30. В.М. Головизнин. Балансно-характеристический метод численного решения уравнений газовой динамики // Докл. РАН, 2005, т.403, № 4, с.459-464;

V.M. Goloviznin. Balansno-xarakteristicheskij metod chislennogo resheniya uravnenij gazovoj dinamiki // Dokl. RAN, 2005, t.403, № 4, c.459-464.

31. Б.В. Рогов. Высокоточная монотонная компактная схема бегущего счета для многомерных уравнений гиперболического типа // ЖВМиМФ, 2013, т.53, № 2, с.264-274;

B.V. Rogov. High-order accurate monotone compact running scheme for multidimensional hyperbolic equations // Comp. Math. and Math. Physics, 2013, v.53, №2, p.205-214.

32. M.D. Bragin, B.V. Rogov. Conservative limiting method for high-order bicompact schemes as applied to systems of hyperbolic equations // Appl. Numer. Math., 2020, v.151, p.229-245.

33. B.V. Rogov. Dispersive and dissipative properties of the fully discrete bicompact schemes of the fourth order of spatial approximation for hyperbolic equations // Appl. Numer. Math., 2019, v.139, p.136-155.

34. П. Роуч. Вычислительная гидродинамика. - М.: Мир, 1980;

P. Rouch. Vychislitelnaya gidrodinamika. - M.: Mir, 1980.

35. А.Г. Куликовский, Н.В. Погорелов, А.Ю. Семенов. Математические вопросы численного решения гиперболических уравнений. - М.: Физматлит, 2001;

A.G. Kulikovskij, N.V. Pogorelov, A.Yu. Semenov. Matematicheskie voprosy` chislennogo resheniya giperbolicheskix uravnenij. - M.: Fizmatlit, 2001.

36. С.К. Годунов. Разностный метод численного расчета разрывных течений гидродинамики // Мат. сборник, 1959, 47 (89), №3, с.271-306;

S.K. Godunov. Raznostnyj metod chislennogo rascheta razryvnykh techenij gidrodinamiki // Mat. sbornik, 1959, 47 (89), №3, s.271-306.

37. В.B. Остапенко. О монотонности разностных схем // Сибирский математический 
журнал, 1998, т.39, №5, с.1111-1126;

V.V. Ostapenko. O monotonnosti raznostnykh sxem // Sibirskij matematicheskij zhurnal, 1998, t.39, №5, s.1111-1126.

38. В.В. Остапенко. О сильной монотонности трёхточечных разностных схем // Сибирский математический журнал, 1998, т.39, №6, с.1357;

V.V. Ostapenko. O silnoj monotonnosti tryokhtochechnykh raznostnykh sxem // Sibirskij matematicheskij zhurnal, 1998, t.39, №6, c.1357.

39. В.В. Остапенко. О сильной монотонности нелинейных разностных схем // ЖВМиМФ, 1998, т.38, №7, c.1170-1185;

V.V. Ostapenko. On the strong monotonicity of nonlinear difference schemes // Comp. Math. and Math. Phys., 1998, v.38, №7, s.1119-1133

40. Р.П. Федоренко. Применение разностных схем высокой точности для численного решения гиперболических уравнений // ЖВМиМФ, 1962, т.2, №6, с.1122-1128;

R.P. Fedorenko. Primenenie raznostnykh sxem vysokoj tochnosti dlya chislennogo resheniya giperbolicheskikh uravnenij // ZhVMiMF, 1962, t.2, №6, s.1122-1128.

41. В.П. Колган. Применение принципа минимальных значений производной к построению конечно-разностных схем для расчета разрывных решений газовой динамики // Ученые записки ЦАГИ, 1972, т.3, № 6, с.68-77;

V.P. Kolgan. Primenenie principa minimalnykh znachenij proizvodnoj k postroeniyu konechno-raznostnykh sxem dlya rascheta razryvnykh reshenij gazovoj dinamiki // Uchenye zapiski CzAGI, 1972, t.3, № 6, s.68-77.

42. B. Van Leer. Towards the ultimate conservative difference scheme II: Monotonicity and conservation combined in a second order scheme // J. of Comp. Physics, 1974, v.14, №361, p.370.

43. B. Van Leer. Towards the ultimate conservative difference schemes. III. Upstream-centered finite-difference schemes for ideal compressible flow // J. of Comp. Physics, 1977, v.23, № 3, p.263-275.

44. B. Van Leer. Towards the ultimate conservative difference scheme V: A second order sequel to Godunov s method // J. of Comp. Physics, 1979, v.32, p.101-136.

45. J.P. Boris, D.L. Book. Flux-corrected transport II: Generalization of the method // J. of Comp. Phys., 1975, v.18, p.248-283.

46. S.T. Zalesak. Fully multidimensional flux-corrected transport algorithms for fluids // J. of Comp. Phys., 1979, v.31, p.335.

47. А.И. Жмакин, А.А. Фурсенко. Об одной монотонной разностной схеме сквозного счета А // ЖВМиМФ, 1980, т.20, №4, с.1021-1031;

A.I. Zhmakin, A.A. Fursenko. On a monotonic shock-capturing difference scheme // U.S.S.R. Comp. Math. and Math. Phys., 1980, v.20, №4, p.218-227.

48. A. Harten, $S$. Osher. Uniformly high-order accurate non-oscillatory schemes. I// SIAM J. Numer. Anal., 1987, v.27, p.279-309.

49. A. Harten, B. Engquist, S. Osher, S.R. Chakravarthy. Uniformly high order accuracy essentially non-oscillatory schemes. III // J. of Comp. Phys., 1987, v.71, p.231-303.

50. A. Harten. On a class of high resolution total-variation-stable finite-difference schemes // SIAM. J. Numer. Anal., 1984, v.21, p.1-23.

51. S. Osher. Riemann solvers, the entropy condition, and difference approximation // SIAM J. Numer. Anal., 1984, v.21, №2, p.217-235.

52. H.G. Yee, R.F. Warming, A. Harten. Implicit total variation diminishing (TVD) schemes 
for steady-state calculations // J. of Comp. Phys., 1985, v.57, p.327-360.

53. A. Harten. ENO schemes with subset resolution // J. of Comp. Phys., 1989, v.83, №2, p.148-184.

54. A. Harten, S. Osher. Uniformly high-order accurate essentially non-oscillatory scheme. I // SIAM J. Numer. Anal., 1987, v.24, №2, p.279-309.

55. D.K. Mao. A treatment of discontinuities in shock-capturing finite difference methods // J. of Comp. Phys., 1991, v.92, №2, p.422-455.

56. H. Choi, J.G. Liu. The reconstruction of upwind fluxes for conservation laws: Its behavior in dynamic and steady state calculations // J. of Comp. Phys., 1998, v.144, №2, p.237-256.

57. К.В. Вязников, В.Ф. Тишкин, А.П. Фаворский. Построение монотонных разностных схем повышенного порядка аппроксимации для систем уравнений гиперболического типа // Матем. мод., 1989, т.1, №5, с.95-120;

K.V. Vyaznikov, V.F. Tishkin, A.P. Favorskij. Postroenie monotonnykh raznostnykh sxem povyshennogo poryadka approksimacii dlya sistem uravnenij giperbolicheskogo tipa // Matem. Mod., 1989, t.1, №5, c.95-120.

58. A. Harten, B. Engquist, S. Osher, S.R. Chakravarthly. Uniformly high-order accurate essentially non-oscillatory scheme. III // J. of Comp. Phys., 1987, v.71, №2, p.231-303.

59. Ch.W. Shu, S. Osher. Efficient implementation of essentially non-oscillatory shock-capturing schemes // J. of Comp. Phys., 1988, v.77, №2, p.439-471.

60. Ch.W. Shu, S. Osher. Efficient implementation of essentially non-oscillatory shock-capturing schemes. II // J. of Comp. Phys., 1989, v.83, №1, p.32-78.

61. X.D. Liu, S. Osher, T. Chan. Weighted essentially non-oscillatory schemes // J. of Comp. Phys., 1994, v.115, p.200.

62. G. Jiang, C.W. Shu. Efficient implementation of weighted ENO schemes // J. of Comp. Phys., 1996, v.126, p.202.

63. M. Dumbser, M. Kaser and J. de la Puente. Arbitrary High Order Finite Volume Schemes for Seismic Wave Propagation on Unstructured Meshes in 2D and 3D. Geophys // Journal Int., 2000, v.6, p.134-165.

64. M. Dumbser. Arbitrary High Order Schemes for the Solution of Hyperbolic Conservation Laws in Complex Domains // Shaker Verlag, Aachen, 2005.

65. M. Dumbser, C.D. Munz. ADER discontinuous Galerkin schemes for aeroacoustics // Comptes Rendus Mecanique, 2005, 333, p.683-687.

66. M. Dumbser, C.D. Munz. Arbitrary High Order Discontinuous Galerkin Schemes / Numerical Methods for Hyperbolic and Kinetic Problems; eds. S. Cordier, T. Goudon, M. Gutnic, E. Sonnendrucker // IRMA series in math. and theoretical physics, EMS Publishing House, 2005, p.295-333.

67. М.Е. Ладонкина, О.А. Неклюдова, В.Ф. Тишкин, В.С. Чеванин. Об одном варианте существенно неосциллирующих разностных схем высокого порядка точности для систем законов сохранения // Матем. мод., 2009, т.21, №11, с.19-32;

M.E. Ladonkina, O.A. Neklyudova, V.F. Tishkin, V.S. Chevanin. A version of essentially nonoscillatory high-order accurate difference schemes for systems of conservation laws // Math. Models and Comp. Simul., 2010, v.2, №3, p.304-316.

68. E.F. Toro. Riemann Solvers and Numerical Methods for Fluid Dynamics: A Practical Introduction. 2nd Edition, Springer, 1999.

69. E.F. Toro, V.A. Titarev. Solution of the generalized Riemann problem for advection-reac- 
tion equations // Proc. Roy. Soc. - London: 2002, p.271-281.

70. E.F. Toro, V.A. Titarev. ADER: Arbitrary high order Godunov approach // J. of Scientific Computing, 2002, № 17(1-4), p.609-618.

71. E.F. Toro, V.A. Titarev. ADER schemes for three-dimensional nonlinear hyperbolic systems // J. of Comp. Phys., 2005, № 204, p.715-736.

72. E.F. Toro, V.A. Titarev. ADER schemes for scalar hyperbolic conservation laws with source terms in three space dimensions // J. of Comp. Phys., 2005, № 202, p.196-215.

73. M. Dumbser. Arbitrary High Order Schemes for the Solution of Hyperbolic Conservation Laws in Complex Domains // Shaker Verlag, Aachen, 2005.

74. M. Dumbser, C.D. Munz. ADER discontinuous Galerkin schemes for aeroacoustics // Comptes Rendus Mecanique, 2005, v.333, p.683-687.

75. M. Dubiner. Spectral methods on triangles and other domains // Journal of Sci. Computing, 1991, № 6, p.345-390.

76. M. Dumbser et al. Quadrature-free non-oscillatory finite volume schemes on unstructured meshes for nonlinear hyperbolic systems // J. of Comp. Phys., 2007, v.221, №2, p.693-723.

77. I. Abalakin, P. Bakhvalov, T. Kozubskaya. Edge-based reconstruction schemes for unstructured tetrahedral meshes // Intern. J. for numer. methods in fluids, 2016, v.81, №6, p.331-356.

78. P. Bakhvalov, T. Kozubskaya. EBR-WENO scheme for solving gas dynamics problems with discontinuities on unstructured meshes // Comp. Fluids, 2017, №157, p.312-324.

79. С.К. Годунов. Разностный метод численного расчета разрывных течений гидродинамики // Мат. сборник, 1959, 47 (89), №3, с.271-306.

S.K. Godunov. Raznostnyj metod chislennogo rascheta razryvnykh techenij gidrodinamiki // Mat. sbornik, 1959, 47 (89), №3, s.271-306.

80. W.H. Teukolsky, S.A. Vetterling. W.T. Flannery. BP «Section 10.1.2. Lax Method», Numerical Recipes: The Art of Scientific Computing (3rd ed.), New York: Cambridge University Press, 2007. [Электронный pecypc] http://apps.nrbook.com/empanel/index.html\# $\mathrm{pg}=1034$. (дата обращения 01.03.2015).

81. P.D. Lax. Weak solutions of nonlinear hyperbolic equations and their numerical computation // Comm. on Pure and Appl. Math., 1954, v.7, №1, p.159-193.

82. В.В. Русанов. Расчет взаимодействия нестационарных ударных волн с препятствиями // ЖВМиМФ, 1961, т.I, №2, с.267-279;

V.V. Rusanov. Raschet vzaimodejstviya nestacionarnykh udarnykh voln s prepyatstviyami // ZhVMiMF, 1961, t.I, №2, s.267-279.

83. A. Kurganov, E. Tadmor. New High-Resolution Central Schemes for Nonlinear Conservation Laws and Convection-Diffusion Equations // J. of Comp. Phys., 2000, № 160, p.241-282.

84. J. Balbas, E. Tadmor, C.C. Wu. Central non-oscillatory schemes for one- and two-dimensional MHD equations // J. of Comp. Phys., 2004, № 201(1), p.261-285.

85. J. Qiu, C.W. Shu. On the construction, comparison, and local characteristic decompositions for high order central WENO schemes // J. of Comp. Phys., 2002, № 183, p.187-209.

86. E. Tadmor, J. Tanner. An adaptive order Godunov type central scheme «Hyperbolic Problems: Theory, Numerics, Applications» // Proc. of the 9th international conference held at CalTech, 2002 Mar. / Hou T., Tadmor E., eds. Springer, 2003, p.871-880.

87. P. Arminjon, M.C. Viallon, A. Madrane. A finite volume extension of the Lax-Friedrichs and Nessyahu-Tadmor schemes for conservation laws on unstructured grids International // J. of Comp. Fluid Dynam., 1997, № 9(1), p.1-22. 
88. S. Karni, E. Kirr et al. Compressible two-phase flows by central and upwind schemes // Math. Mod. and Num. Anal., 2004, 38(3), p.477-493.

89. P. Woodward, Ph. Colella. The numerical simulation of two-dimensional fluid flow with strong shocks // J. of Comp. Phys., 1984, v.54, №1, p.115-173.

90. Ph. Colella, P.R. Woodward. The piecewise parabolic method (PPM) for gas-dynamical simulations // J. of Comp. Phys., 1984, v.54, №1, p.174-201.

91. R.H. Cohen, A.A. Mirin. ASCI turbulence and instability modeling using the piecewise parabolic method. Lawrence Livermore National Laboratory, UCRL-TB-125580, Rev.4, 1999. [Электронный ресурс] https://computation.llnl.gov/casc/asciturb/asciturb.html. (дата обращения 01.03.2015).

92. P. Colella, L.F. Henderson. The von Neumann paradox for the diffraction of weak shock waves // Journal Fluid Mechanics, 1990, v.213, p.71-94.

93. M.J. Berger, P. Colella. Local adaptive mesh refinement for shock hydrodynamics // J. of Comp. Phys., 1989, v.82, №1, p.64-84.

94. Ю.В.Янилкин, В.П.Стаценко, В.И. Козлов. Математическое моделирование турбулентного перемешивания в сжимаемых средах. - Саров: РФЯЦ ВНИИЭФ, 2009;

Yu.V. Yanilkin, V.P. Stacenko, V.I. Kozlov. Matematicheskoe modelirovanie turbulentnogo peremeshivaniya v szhimaemykh sredax. - Sarov: RFYaCz VNIIEF, 2009.

95. W.J. Rider. Reconsidering remap methods // Intern J. for Numerical Methods in Fluids, 2014, v.76, p.587.

96. М.В. Попов, С.Д. Устюгов. Кусочно-параболический метод на локальном шаблоне для задач газовой динамики // ЖВМиМФ, 2007, т.47, №12, с.2055-2075;

M.V. Popov, S.D. Ustyugov. Piecewise parabolic method on local stencil for gasdynamic simulations // Comp. Math. and Math. Phys., 2007, v.47, №12, p.1970-1989.

97. М.В. Попов, С.Д. Устюгов. Кусочно-параболический метод на локальном шаблоне для идеальной магнитной газодинамики // ЖВМиМФ, 2008, т.48, №3, с.505-528.

M.V. Popov, S.D. Ustyugov. Piecewise parabolic method on a local stencil for ideal magnetohydrodynamics // Comp. Math. and Math. Phys., 2008, v.48, №3, p.477-499

98. S.D. Ustyugov, M.V. Popov, A.G. Kritsuk, M.L. Norman. Piecewise Parabolic Method on a Local Stencil for Magnetized Supersonic Turbulence Simulation // J. of Comp. Phys., 2009, № 228, p.7614-7633.

99. A. Suresh, H.T. Huynh. Accurate Monotonicity-Preserving Schemes with Runge-Kutta Time Stepping // J. of Comp. Phys., 1997, № 136, p.83.

100. W.J. Rider et al. Accurate monotonicity- and extrema-preserving methods through adaptive nonlinear hybridizations // J. of Comp. Phys., 2007, № 225, p.1827

101. [Электронный ресурс] http://www.astro.virginia.edu/VITA/ATHENA/bow-shock.html. (дата обращения 01.03.2015).

102. W.H. Reed and T.R. Hill. Triangular mesh methods for the neutron transport equation // Los Alamos Scientific Laboratory Report LA-UR-73-79, 1973, USA. doi: https://www. osti.gov/servlets/purl/4491151

103. Proc. ECCOMAS Thematic Conference: European Conf. on High Order Nonlinear Numerical Methods for Evolutionary PDEs: Theory and Appl. HONOM, 2017, 27.03-31.03.

104. Cockburn and $C-W$. Shu. The Runge-Kutta discontinuous Galerkin method for conservation laws V: Multidimensional systems // J. Comp. Phys., 1998, v.141, p.199-224.

105. А.В. Волков. Особенности применения метода Галеркина к решению пространст- 
венных уравнений Навье-Стокса на неструктурированных гексаэдральных сетках // Ученые записки ЦАГИ, 2009, № 6, т. XL;

A.V. Volkov. Osobennosti primeneniya metoda Galerkina k resheniyu prostranstvennykh uravnenij Navie-Stoksa na nestrukturirovannykh geksaedralnykh setkakh // Uchenye zapiski CzAGI, 2009, № 6, t.XL.

106. R. Nastase and D.J. Mavriplis. High-order discontinuous Galerkin methods using an hpmultigrid approach // J. Comp. Phys., 2006, 213, p.330-357.

107. H. Luo, J. D. Baum and R. Löhner. Fast p-multigrid discontinuous Galerkin method for compressible flow at all speeds// AIAA J., 2008, №46, p.635-652.

108. F. Bassi and S. Rebay. Numerical evaluation of two discontinuous Galerkin methods for the compressible Navier-Stokes equations // Int. J. Numer. Meth. Fluids, 2002, v.40, p.197-207.

109. D.N. Arnold, F. Brezzi, B. Cockburn, L.D. Marini. Unified analysis of discontinuous Galerkin methods for elliptic problems // SIAM J. on Numer. Analysis, 2002, v.29, p.1749-1779.

110. С.М. Босняков, С.В. Михайлов, В.Ю. Подаруев, А.И. Трошин. Нестационарный разрывный метод Галеркина высокого порядка точности для моделирования турбулентных течений // Матем. моделир., 2018, т.30, №5, с.37-56.

S.M. Bosnyakov, S.V. Mikhailov, V.Yu. Podaruev, A.I. Troshin. Nestacionarnyj razryvnyj metod Galerkina vysokogo poryadka tochnosti dlya modelirovaniya turbulentnykh techenij // Matem. Mod., 2018, t.30, №5, s.37-56.

111. M.M. Krasnov et al. Numerical solution of the Navier-Stokes equations by discontinuous Galerkin method // J. of Physics: Conf. Ser., 2017, №1, v.815.

112. М.М. Краснов, П.А. Кучугов, М.Е. Ладонкина, В.Ф. Тишкин. Разрывный метод Галеркина на трехмерных тетраэдральных сетках. Использование операторного метода программирования // Матем. моделирование, 2017, т.29, № 2, с.3-22;

M.M. Krasnov, P.A. Kuchugov, M.E. Ladonkina, V.F. Tishkin. Discontinuous Galerkin method on three-dimensional tetrahedral grids: Using the operator programming method // Math. Models and Comp. Simul., 2017, v.9, №5, p.529-543.

113. М.М. Краснов, М.Е. Ладонкина. Разрывный метод Галёркина на трёхмерных тетраэдральных сетках. Применение шаблонного метапрограммирования языка $\mathrm{C}++/ /$ Программирование, 2017, №3, с.41-53;

M.M. Krasnov, M.E. Ladonkina. Discontinuous Galerkin method on three-dimensional tetrahedral grids. The use of template metaprogramming of the $\mathrm{C}++$ language // Programming and Computer Software, 2017, v.43, № 3, p.172-183.

114. М.Е. Ладонкина, О.А. Неклюдова, В.Ф. Тишкин. Использование разрывного метода Галёркина при решении задач гидродинамики // Мат. модел., 2014, т.26, №1, с.17-32; M.E. Ladonkina, O.A. Neklyudova, V.F. Tishkin. Application of the RKDG method for gas dynamics problems // Math. Models and Comp. Simul., 2014, v.6, №4, p.397-407.

115. K. Yasue, M. Furudate, N. Ohnishi and K. Sawada. Implicit discontinuous Galerkin method for RANS simulation utilizing pointwise relaxation algorithm //Communications in Computational Physics, 2010, v.7, №.3, p.510-533.

116. Cockburn. An Introduction to the Discontinuous Galerkin Method for Convection Dominated Problems, Advanced Numerical Approximation of Nonlinear Hyperbolic Equations // Lecture Notes in Mathematics, 1998, v.1697, p.151-268.

117. L. Krivodonova. Limiters for high-order discontinuous Galerkin methods // J. of Comp. 
Phys., 2007, 226, №1, p.276-296.

118. C.-W. Shu. High order WENO and DG methods for time-dependent convection-dominated PDEs: A brief survey of several recent developments // J. of Comp. Phys., 2016, v.316, p.598- 613.

119. H. Luo, J.D. Baum, R.A. Lohner. Hermite WENO-based limiter for discontinuous Galerkin method on unstructured grids // J. of Comp. Phys., 2007, v.225, I. 1, p.686-713.

120. J. Zhu, X. Zhong, C.-W. Shu, J. Qiu. Runge-Kutta discontinuous Galerkin method using a new type of WENO limiters on unstructured meshes // J. of Comp. Phys., 2013, v.248, p.200-220.

121. M. Dumbser. Arbitrary high order PNPM schemes on unstructured meshes for the compressible Navier-Stokes equations // Computers \&Fluids, 2010, v.39, I.1, p.60-76.

122. J. Peraire, P.-O. Persson. Adaptive High-Order Methods in Computational Fluid Dynamics, V.2 of Advances in CFD, chap.5 - High-Order Discontinuous Galerkin Methods for CFD // World Scientic Publishing Co., 2011.

123. А.В. Волков, С.В. Ляпунов. Монотонизация метода конечного элемента в задачах газовой динамики // Уч. записки ЦАГИ, 2009, т.XL, № 4, c.15-27;

A.V. Volkov, C.V. Lyapunov. Monotonizaciya metoda konechnogo elementa v zadachax gazovoj dinamiki // Uch. zapiski CzAGI, 2009, t.XL, № 4, s.15-27.

124. М.Е. Ладонкина, О.А. Неклюдова, В.Ф. Тишкин. Построение лимитера для разрывного метода Галеркина на основе усреднения решения // Матем. модел., 2018, т.30, №5, c.99-116.

M.E. Ladonkina, O.A. Neklyudova, V.F. Tishkin. Constructing a Limiter Based on Averaging the Solutions for the Discontinuous Galerkin Method // Math. Models \& Comp. Simul., 2019, v.11, p.61-73.

125. T. Haga, K. Sawada. An improved slope limiter for high-order spectral volume methods solving the 3D compressible Euler equations, 2009.

126. Cockburn, C.-W. Shu. The local discontinuous Galerkin method for time dependent convection diffusion system // SIAM J. Numer. Anal., 1998, v.35, №6, p.2440-2463.

127. В.В. Власенко, А.В. Волков, А.И. Трошин. Выбор метода аппроксимации вязких членов в методе Галеркина с разрывными базисными функциями // Ученые записки ЦАГИ, 2013, т.XLIV, № 3;

V.V. Vlasenko, A.V. Volkov, A.I. Troshin. Vybor metoda approksimacii vyazkikh chlenov v metode Galerkina s razryvnymi bazisnymi funkciyami // Uchenye zapiski CzAGI, 2013, t.XLIV, № 3 .

128. S. Gottlieb, C.-W. Shu, E. Tadmor. Strong stability-preserving high-order time discretization methods // SIAM Review, 2001, v.43, №1, p.89-112.

129. J. Spiteri Raymond, J. Ruuth Steven. A New Class of Optimal High-Order Strong Stability-Preserving Time Discretization Methods // SIAM J. Numer. Anal., 2002, v.40, №2, p.469-491.

130. P. Rasetarinera, M.Y. Hussaini. An efficient implicit discontinuous spectral Galerkin method // J. Comp. Phys., 2001, v.172, p.718-738.

131. R. Hartmann. Adaptive discontinuous Galerkin methods with shock-capturing for the compressible Navier-Stokes equations // Int. J. Numer. Meth. Fluids, 2006, №51, p.1131-1156.

132. R. Hartmann, P. Houston. Symmetric interior penalty DG methods for the compressible Navier-Stokes equations I: Method formulation // Int. J. Numer. Anal. Model., 2006, №3, p.1-20. 
133. V. Dolejšsi. Semi-implicit interior penalty discontinuous Galerkin methods for viscous compressible flows // Commun. Comp. Phys., 2008, v.4, p.231-274.

134. K. Yasue, M. Furudate, N. Ohnishi, K. Sawada. Implicit discontinuous Galerkin method for RANS simulation utilizing pointwise relaxation algorithm // Commun. Comp. Physics, 2010, v.7, №3, p.510-533.

135. A. Jameson, S. Yoon. Lower-upper implicit schemes with multiple grids for the Euler equations // AIAA J., 1987, v.25, p.929-935.

136. A. Klockner, T. Warburton, J.S. Hesthaven. Nodal discontinuous Galerkin methods on graphics processors // J. of Comp. Phys., 2009, 228, №21, p.7863-7882.

137. J. Lou, Y. Xia, L. Luo, H. Luo, J.R. Edwards, F. Mueller. OpenACC-based GPU Acceleration of a p-multigrid Discontinuous Galerkin Method for Compressible Flows on 3D Unstructured Grids // 53rd AIAA Aerospace Sciences Meeting, AIAA SciTech Forum, (AIAA 2015-0822).

138. J. Chan, et al. GPU-accelerated discontinuous Galerkin methods on hybrid meshes // J. of Comp. Phys., 2015, p.318.

139. М.М. Краснов. Операторная библиотека для решения трехмерных сеточных задач математической физики с использованием графических плат с архитектурой CUDA // Матем. Мод., 2015, т.27, № 3, с.109-120;

M.M. Krasnov. Operatornaya biblioteka dlya resheniya trexmernykh setochnykh zadach matematicheskoj fiziki s ispolzovaniem graficheskix plat s arxitekturoj CUDA // Matem. Mod., 2015, t.27, № 3, s.109-120.

140. М.М. Краснов. Параллельный алгоритм вычисления точек гиперплоскости фронта вычислений // ЖВМиМФ, 2015, тю55, №1, с.145-152.

M.M. Krasnov. Parallel algorithm for computing points on a computation front hyperplane Comp. Math. Math. Phys., 55:1 (2015), 140-147

141. Ph. Mocz et al. A discontinuous Galerkin method for solving the fluid and MHD equations in astrophysical simulations // Mon. Not. R. Astron. Soc., 2014, v.437, p.397-414.

142. М.М. Баско. Метод искусственной вязкости расчета одномерных течений // ЖВМ и МФ, 1990, т.30, № 4, с.601-610;

M.M. Basko. The method of artificial viscosity for computing one-dimensional flows // U.S.S.R. Comp. Math. and Math. Phys., 1990, v.30, №2, p.176-182.

143. M.P. Arminjon, A. Dervieux. Construction of TVD-like artificial viscosities on 2-dimensional arbitrary FEM grids// INRIA Research Report 1111, 1989.

144. W.D. Schultz. Two-dimensional Lagrangian hydrodynamic difference equations // Methods of Computational Physics. - NewYork: Academic Press, 1964, v.3.

145. И.В. Попов, И.В. Фрязинов. Адаптивная искусственная вязкость для многомерной газовой динамики в эйлеровых переменных в декартовых координатах // Матем. Моделирование, 2010, т.22, №1, с.32-45;

I.V. Popov, I.V. Fryazinov Adaptive artificial viscosity for multidimensional gas dynamics for Euler variables in Cartesian coordinates // Math. Models and Comp. Simul., 2010, v.2, №4, p.429-442

146. Е.В. Ступоченко, С.А. Лосев, А.И. Осипов. Релаксационные процессы в ударных волнах. - М.: Наука, 1965.

E.V. Stupochenko, S.A. Losev, A.I. Osipov. Relaksacionnye processyv udarnykh volnakh. - 
M.: Nauka, 1965.

147. Б.Н. Четверушкин. Кинетически-согласованные схемы в газовой динамике. - М.: Изд-во МГУ, 1999;

B.N. Chetverushkin. Kineticheski-soglasovannye skhemy v gazovoj dinamike. - M.: Izdvo MGU, 1999.

148. А.С. Болдарев, В.А. Гасилов, О.Г. Ольховская. К решению гиперболических уравнений на неструктурированных сетках // Матем. моделирование, 1996, т.8, №3, с.51-78; A.S. Boldarev, V.A. Gasilov, O.G. Olkhovskaya. K resheniyu giperbolicheskix uravnenij na nestrukturirovannykh setkakh // Matem. Mod., 1996, t.8, №3, s.51-78.

149. В.Ф. Куропатенко. Метод построения разностных схем для численного интегрирования уравнений газодинамики // Известия ВУЗов. Математика, 1962, № 3, с.75-83; V.F. Kuropatenko. Metod postroeniya raznostnykh skhem dlya chislennogo integrirovaniya uravnenij gazodinamiki // Izvestiya VUZov. Matematika, 1962, № 3, c.75-83.

150. R.B. Christensen. Godunov methods on a staggered mesh an improved artificial viscosity // Lawrence Livermore Laboratory report, UCRL-JC-105269, 1991.

151. O.V. Diyankov, I.V. Glazyrin, S.V. Koshelev. MAG - two-dimensional resistive MHD -code using an arbitrary moving coordinate system // Comp. Phys. Commun, 1997, v.106, p.76-94.

152. Ю.А. Повещенко и др. Об одной двухслойной полностью консервативной разностной схеме газовой динамики в эйлеровых переменных с адаптивной регуляризацией решения // Препринты ИПМ им. М.В. Келдыша, 2019, № 14, 23 с. doi:10.20948/prepr-2019-14; Yu.A. Poveshhenko $i d r$. Ob odnoj dvukhslojnoj polnostyu konservativnoj raznostnoj skheme gazovoj dinamiki $\mathrm{v}$ ejlerovykh peremennykh $\mathrm{s}$ adaptivnoj regulyarizaciej resheniya // Preprinty IPM im. M.V. Keldysha, 2019, № 14, 23 s.

153. S.R. Kennon, G.S. Dulikravich. A posteriori optimization of computational grid // AIAA85-0483, 1985.

154. Н.А. Дарьин, В.И. Мажукин, А.А. Самарский. Конечно-разностный метод решения уравнений газовой динамики с использованием адаптивных сеток, динамически связанных с решением // ЖВМиМФ, 1988, т.28, № 8, с.1210-1225;

N.A. Darin, V.I. Mazhukin, A.A. Samarskii. A finite-difference method for solving the equations of gas dynamics using adaptive nets which are dynamically associated with the solution // U.S.S.R. Comp. Math. and Math. Phys., 1988, v.28, №4, p.164-174

155. G. Beckett, J.A. Mackenzie, A. Ramage, D.M. Sloan. On the numerical solution of onedimensional PDEs using adaptive methods based on equidistribution // J. Comp. Phys. 2001, № 167, p.372-392.

156. K.J. Fidkowski, D.L. Darmofal. Output-Based Error Estimation and Mesh Adaptation in Computational Fluid Dynamics: Overview and Recent Results // AIAA 2009-1303.

157. W. Huang, R.D. Russell. Adaptive Moving Mesh Methods // Springer Science+Business Media, LLC, 2011.

158. D.F. Hawken, J.J. Gottlieb, J.S. Hansen. Review of some adaptive node-movement techniques in finite element and finite-difference solutions of partial differential equations // J. Comp. Phys., 1991, №95, p.254-302.

159. J.F. Dannenhoffer. A comparison of adaptive-grid redistribution and embedding for steady transonic flows // Intern. J. for Numerical Methods in Engineering, 1991, №32, p.653-663.

160. M. Berger, J. Oliger. Adaptive mesh refinement for hyperbolic partial differential equations // J. Comp. Phys., 1984, № 53, p.484-512. 
161. R. Klein. Star formation with 3-D adaptive mesh refinement: the collapse and fragmentation of molecular clouds // J. of Comp. and App. Math., 2011, v.16, № 109(1-2), p.123-152. doi:10.1016/S0377-0427(99)00156-9

162. Kumar Khattri Sanjay. Grid Generation and Adaptation by Functionals // Computational \& Applied Mathematics, 2007. v.26, № 2, p.235-249.

163. А.Е. Луикий, А.В. Пленкин. Численное моделирование на адаптивных сетках взаимодействия ударных волн с пограничными слоями // Препр. ИПМ им. М.В.Келдыша, 2016, № 136, 15 c. doi:10.20948/prepr-2016-136;

A.E. Luczkij, A.V. Plenkin. Chislennoe modelirovanie na adaptivnykh setkakh vzaimodejstviya udarnykh voln s pogranichnymi sloyami // Prepr. IPM im. M.V.Keldysha, 2016, №136, 15s

164. А.Л. Афендиков и др. Алгоритм многоуровневой адаптации сеток по критериям на основе вейвлет-анализа для задач газовой динамики // Препринты ИПМ им. М.В. Келдыша, 2015, № 97, 22 с.;

A.L. Afendikov $i$ dr. Algoritm mnogourovnevoj adaptacii setok po kriteriyam na osnove vejvlet-analiza dlya zadach gazovoj dinamiki // Preprinty` IPM im. M.V. Keldysha, 2015, № 97, 22 s.

165. G. Warren, W.K. Anderson, J. Thomas, S. Krist. Grid convergence for adaptive methods // InAIAA 10th Comp. Fluid Dynamics Conference, 1991.

166. K.G. Powell, P.L. Roe, J. Quirk. Adaptive-Mesh Algorithms for Computational Fluid Dynamics // In: Hussaini M.Y., Kumar A., Salas M.D. (eds) Algorithmic Trends in Computational Fluid Dynamics. ICASE/NASA LaRC Series. Springer, New York, NY, 1993.

167. O. Antepara, O. Lehmkuhl, J. Chiva, R. Borrell. Parallel Adaptive Mesh Refinement Simulation of the Flow Around a Square Cylinder at $R e=22000$ // Procedia Eng., 2013, №61, p.246-250.

168. G. Li, X. Fuamp; F. Wang. High-resolution multi-code implementation of unsteady NavierStokes flow solver based on paralleled overset adaptive mesh refinement and high-order low -dissipation hybrid schemes // Int. J. of Comp. Fluid Dyn., 2017, doi: 10.1080/ 10618562. 2017. 1387251.

169. A. Thornber, J. Griffond, O. Poujade et al. Late-time growth rate, mixing, and anisotropy in multimode narrowband Richtmyer-Meshkov instability: The $\theta$-group collaboration// Physics of Fluids, 2017, v.29, №10, p.105107.

170. G. Dimonte, D.L. Youngs, A. Dimits, et al. A comparative study of the turbulent Rayleigh-Taylor instability using high resolution three dimensional numerical simulations: The alpha-group collaboration // Physics of Fluids, 2004, v.16, №5, p.1668-1693.

Поступила в редакцию 16.04.2020

После доработки 16.04.2020

Принята к публикации 08.06.2020 